\title{
NEAR-LIMIT DROP DEFORMATION AND SECONDARY BREAKUP
}

\author{
L.-P. Hsiang and G. M. FaETh $\dagger$ \\ Department of Aerospace Engineering, University of Michigan, Ann Arbor, MI 48109-2140, U.S.A.
}

(Received 29 January 1992; in revised form 11 May 1992)

\begin{abstract}
The properties of drop deformation and secondary breakup were observed for shock wave initiated disturbances in air at normal temperature and pressure. Test liquids included water, glycerol solutions, $n$-heptane, ethyl alcohol and mercury to yield Weber numbers (We) of $0.5-1000$, Ohnesorge numbers $(\mathrm{Oh})$ of $0.0006-4$, liquid/gas density ratios of 580-12,000 and Reynolds numbers (Re) of $300-16,000$. Measurements included pulsed shadowgraphy and holography to find drop deformation properties prior to breakup, as well as drop size distributions after breakup. Drop deformation and breakup regimes were identified in terms of $\mathrm{We}$ and $\mathrm{Oh}$ : regimes at low $\mathrm{Oh}$ include no deformation, nonoscillatory deformation, oscillatory deformation, bag breakup, multimode breakup and shear breakup as We is increased. However, most of these regimes occur at higher We when $\mathrm{Oh}$ values are increased, with no breakup observed for $\mathrm{Oh}>4$ over the present test range. Unified temporal scaling of deformation and breakup processes was observed in terms of a characteristic breakup time that largely was a function of $\mathrm{Oh}$. Prior to breakup, the drag coefficient evolved from the properties of spheres to those of thin disks as drop deformation progressed. The drop size distribution after breakup satisfied Simmons' universal root normal distribution function for the bag and multimode breakup regimes and could be characterized by the Sauter mean diameter (SMD) alone. Drop sizes after shear breakup, however, did not satisfy this distribution function due to the distorting effect of the core or drop-generating drop. Nevertheless, the SMD after secondary breakup could be correlated in terms of a characteristic liquid boundary layer thickness for all breakup regimes, similar to recent results for nonturbulent primary breakup. Drop properties after secondary breakup suggest that both reduced drop sizes and reduced relative velocities play a role in ending the secondary breakup process.
\end{abstract}

Key Words: sprays, atomization, drop breakup.

\section{INTRODUCTION}

The secondary breakup of drops is an important multiphase flow process with applications to liquid atomization, dispersed multiphase flow, combustion instability of sprays, heterogeneous detonations of gas/liquid mixtures, the properties of rain, and interactions between high-speed aircraft and raindrops, among others. In particular, recent studies of the structure of dense pressure-atomized sprays [see Ruff et al. (1992) and Faeth (1990) and references cited therein] confirm the conventional view of liquid atomization with primary breakup at the liquid surface followed by secondary breakup. It also was found that secondary breakup can control mixing rates of dense sprays in some instances, much like drop vaporization often controls mixing rates of dilute sprays. Finally, recent studies of primary breakup of both nonturbulent and turbulent liquids show that primary breakup intrinsically yields drops that are unstable to near-limit secondary breakup (Wu et al. 1991, 1992). Motivated by these observations, the objectives of the present investigation were to study drop deformation and breakup for well-defined shock wave disturbances (yielding a step change in the relative velocity of a drop) at conditions near the onset of secondary breakup. Issues considered include the required flow conditions, dynamics and the outcomes of drop deformation and breakup.

Due to numerous applications, secondary breakup has received significant attention in the past. Giffen \& Muraszew (1953) and Hinze (1955) reviewed early work in the field; therefore, the following discussion will be limited to more recent studies. The definition of the onset of breakup, breakup dynamics and the outcome of breakup will be considered, in turn. Most earlier work at least has touched on the definition and conditions for the onset of various breakup regimes. The breakup regime observed at the onset of secondary breakup has been termed bag breakup: it 
involves deflection of the drop into a thin disk normal to the flow direction, followed by deformation of the center of the disk into a thin balloon-like structure extending in the downstream direction, both of which subsequently divide into drops [see Hinze (1955), Hanson et al. (1963), Gel'fand et al. (1974), Krzeczkowski (1980), Ranger \& Nicholls (1969), Reinecke \& McKay (1969), Reinecke \& Waldman (1970) and Wierzba \& Takayama (1988) for photographs of all the breakup regimes discussed here]. The shear breakup regime is observed at higher relative velocities: it involves deflection of the periphery of the disk in the downstream direction, rather than the center, and stripping of drops from the periphery of the disk. The transition between the bag and shear breakup regimes involves complex breakup processes, with portions of this regime termed parachute breakup, chaotic breakup, bag-jet breakup, transition breakup etc. (Borisov et al. 1981; Krzeczkowski 1980); this regime will be denoted the multimode breakup regime in the following. A complex breakup mechanism also has been observed at very large relative velocities, which is called the catastrophic breakup regime (Reinecke \& McKay 1969; Reinecke \& Waldman 1970).

Existing observations of secondary breakup have generally involved liquid/gas density ratios, $\rho_{\mathrm{L}} / \rho_{\mathrm{G}}>500$ and Reynolds numbers, $\operatorname{Re}=\rho_{\mathrm{G}} d_{0} u_{0} / \mu_{\mathrm{G}}>100$, where $d_{0}$ and $u_{0}$ denote the original drop diameter and relative velocity and $\mu_{\mathrm{G}}$ is the gas viscosity. For these conditions, Hinze (1955) has shown that transitions between breakup regimes largely are functions of the Weber number, $\mathrm{We}=\rho_{\mathrm{G}} d_{0} u_{0}^{2} / \sigma$, and the Ohnesorge number, $\mathrm{Oh}=\mu_{\mathrm{L}} /\left(\rho_{\mathrm{L}} d_{0} \sigma\right)^{1 / 2}$, where $\sigma=$ surface tension and $\mu_{\mathrm{L}}=$ liquid viscosity, which are measures of the ratios of drag and liquid viscous forces to surface tension forces, respectively. Hinze (1955) found that progressively larger disturbances (i.e. larger We) were required for the onset of breakup as Oh increased, because viscous forces in the liquid tend to inhibit drop deformation (which is the first step in the breakup process) at large Oh. In fact, viscous forces essentially suppressed secondary breakup for the available range of We, for Oh $>2$ (Hinze 1955). Among others, Loparev (1975) showed that the properties of the disturbances also affected the onset of breakup, with more slowly applied disturbances requiring larger values of We for breakup at a particular value of Oh: subsequent considerations will be limited to shock wave disturbances to avoid this complication. Borisov et al. (1981) proposed an alternative breakup regime map in terms of We and Re, considering both the bag and shear breakup regimes, which is best suited to conditions where $\mathrm{Oh} \ll 1$. Krzeczkowski (1980) extended the breakup regime map of Hinze (1955) to locate transitions to the bag, bag-jet, multimode (which Krzeczkowski called transition breakup) and shear breakup regimes as a function of We and $\mathrm{Oh}$. Nevertheless, in spite of its importance for initiating breakup, conditions for the onset of drop deformation and the definition of deformation processes have not received much attention.

Another aspect of secondary breakup that has been studied is the time required to complete breakup. Liang et al. (1988) summarized past measurements of breakup times, including the findings of Simpkins \& Bales (1972) and Ranger \& Nicholls (1969) for shear breakup and those of Reinecke \& McKay (1969) and Reinecke \& Waldman (1970) for catastrophic breakup-all for shock wave disturbances at large $\rho_{\mathrm{L}} / \rho_{\mathrm{G}}$ and low $\mathrm{Oh}$. For these conditions, breakup times could be normalized by a characteristic breakup time, $t^{*}=d_{0}\left(\rho_{\mathrm{L}} / \rho_{\mathrm{G}}\right)^{1 / 2} / u_{0}$, finding that the normalized breakup time does not vary greatly over the large range of We that includes both the shear and catastrophic breakup regimes. However, results near the onset of secondary breakup, within the bag breakup regime, have not been studied very much in spite of the importance of these near-limit conditions to processes within practical sprays (Ruff et al. 1992).

The deformation properties of drops prior to secondary breakup due to shock wave disturbances have been studied for large $\rho_{\mathrm{L}} / \rho_{\mathrm{G}}$ and $\mathrm{Oh}<0.1$. Wierzba \& Takayama (1988) summarized past work in this area, which included the results of Ranger \& Nicholls (1969) and those of Reinecke \& McKay (1969) and Reinecke \& Waldman (1970) for shear and catastrophic breakup, as well as their own measurements of deformation prior to shear breakup. They found that deformation scales in terms of $t^{*}$, although in contrast to breakup times, the behavior of deformation during shear breakup differs somewhat from catastrophic breakup. Additionally, they highlighted problems of interpreting shadowgraph photographs of breakup processes and suggested the use of holography instead. Similar to breakup times, however, drop deformation within the bag and transition breakup regimes have not received much attention.

Finally, due to the problems of observing drops after seconday breakup there is very little information available about the outcome of secondary breakup, even though this information is 
vital for understanding the structure of dense sprays (Faeth 1990). An exception is some limited results reported by Gel'fand $e t$ al. (1974) for the bag breakup regime. A bimodal distribution was observed with small drops resulting from breakup of the bag and a group of larger drops associated with breakup of the liquid ring at the base of the bag. However, this information is too limited to provide general guidance about drop sizes produced by secondary breakup.

The preceding review indicates that there are several gaps in the literature concerning secondary breakup. In particular, conditions for the onset of various breakup regimes have been defined reasonably well by Krzeczkowski (1980) but analogous deformation regimes have not been defined, particulary at high $\mathrm{Oh}$ where liquid viscosity effects are important. Breakup times and drop deformation have been studied as well (Ranger \& Nicholls 1969; Reinecke \& McKay 1969; Reinecke \& Waldman 1970), however, available information is limited for the near-limit bag and transition breakup regimes that are important for drop breakup in dense sprays. Finally, measurement problems have limited information about the outcome of secondary breakup in spite of the importance of this breakup property. Thus, the objectives of the present investigation were to extend the earlier work to provide measurements of the onset of various deformation and breakup regimes, the evolution of breakup processes, and the resulting drop sizes after secondary breakup. Phenomenological descriptions of these processes were used to help interpret the data. Measurements emphasized conditions near the onset of breakup where past information is very limited even though this region is particularly important for understanding the structure of dense sprays. The measurements involved pulsed shadowgraph photography and holography, the latter being particularly useful for finding drop sizes after secondary breakup. The study was limited to conditions similar to those treated by Hinze (1955) and Krzeczkowski (1980), which are representative of sprays near atmospheric pressure conditions: $\rho_{\mathrm{L}} / \rho_{\mathrm{G}}>500$ and $\operatorname{Re}>100$. Shock wave disturbances were considered with water, $n$-heptane, ethyl alcohol, mercury and various glycerol mixtures used as test liquids in order to study effects of liquid phase properties.

The paper begins with a discussion of the experimental methods. Results are then considered, treating breakup regimes, breakup times, drop deformation, drop drag and drop sizes after breakup, in turn.

\section{EXPERIMENTAL METHODS}

\subsection{Apparatus}

A shock tube with the driven section open to the atmosphere, similar to Ranger \& Nicholls (1969), was used to generate shock wave disturbances. The driver section was pressurized with air and was round with i.d. $=75 \mathrm{~mm}$ and a length of $3.1 \mathrm{~m}$. The driven section had a rectangular interior cross section ( $38 \mathrm{~mm}$ wide $\times 64 \mathrm{~mm}$ high) to facilitate visualization of the flow at the test location. A transition section, with the shock tube diaphragm at its downstream end, provided a gradual evolution from the round driver section to the rectangular driven section. The driven section was $6.7 \mathrm{~mm}$ long with the test location $4.0 \mathrm{~m}$ from the downstream end. This arrangement provided test times of $17-21 \mathrm{~ms}$ in the uniform flow region between the shock wave passing the test location and the subsequent arrival of disturbances from the contact surface and reflections from the ends of the shock tube. Test conditions involved relatively weak shock waves having shock Mach numbers of 1.01-1.24; therefore, thin Mylar film (having thicknesses of 19, 25 and $38 \mu \mathrm{m}$ ) was used for the diaphragm between the driver and driven sections of the shock tube. The Mylar film diaphragm was ruptured to initiate operation of the shock tube by heating a fine resistance wire mounted on the film: this provided a clean break of the diaphragm that was otherwise problematical due to small pressure differences across the diaphragm because the shock waves were weak.

The strength of the shock waves was monitored by two piezoelectric pressure transducers (PCB Piezotronics Inc., model 101A05) mounted 660 and $310 \mathrm{~mm}$ upstream of the test location. The outputs of these transducers were recorded using a digital oscilloscope (LeCroy, model 9400A). The time of passage of the wave between the two transducers provided the shock Mach number (whose properties were checked for consistency using the pressure ratio across the wave). The time required 
to break the diaphragm with the heater wire was not very reproducible; therefore, the pressure signals were used to synchronize data accumulation from the experiment.

The drop generator system involved: a vibrating capillary tube, similar to Dabora (1967), to generate a stream of drops; and a drop selection system, similar to Sangiovanni \& Kestin (1977), to vary the spacing between drops. The test liquid was placed in a reservoir and pressurized with air so that it flowed to a vibrator chamber and then through a capillary tube $(20,23$ or $25 \mathrm{G}$ needles, $12 \mathrm{~mm}$ long, depending on the test condition). The upper end of the vibrator chamber was mechanically attached to a speaker (Realistic, model 40-1319) which, in turn, was driven by a signal generator (BK-Precision, model 3020). By varying the liquid flow rate and the frequency of vibration, a uniformly spaced stream of monodisperse drops was generated by Rayleigh breakup. This drop stream passed through $6 \mathrm{~mm}$ dia holes in the top and bottom of driven section, crossing the central plane of the driven section at the test location. Quartz windows $(25 \mathrm{~mm}$ high $\times 305 \mathrm{~mm}$ long and mounted flush with the inside walls of the driven section) allowed observation of the interaction between the uniform gas flow behind the shock wave and the drop stream.

The separation between drops at the center of the test location due to operation of the vibrating capillary tube alone was 3-4 mm, which was sufficient to allow observation of drop deformation in the early stages of bag and multimode breakup, as well as the shear breakup process, without interactions between drops. However, it was necessary to increase the spacing between drops to observe the later stages and outcomes of bag and multimode breakup. This was accomplished using the approach of Sangiovanni \& Kestin (1977), by charging every other drop in the flow and electrostatically deflecting the charged drops out of the drop stream crossing the shock tube. This yielded a drop spacing of roughly $7 \mathrm{~mm}$, which assured the presence of drops in the region of observation when film records were made while minimizing interactions between drops.

\subsection{Instrumentation}

\subsubsection{Pulsed shadowgraphy}

Drops were observed in two ways: pulsed shadowgraph photographs and motion pictures to observe the overall dynamics of breakup; and single pulse holography to observe the outcome of breakup. Initial work involved pulsed shadowgraph photography using a Xenon Corp. Micropulser (model 457A, $10 \mathrm{~J}$ optical power per pulse with a pulse duration of roughly $1 \mu \mathrm{s}$ ). The lamp output was collimated and directed through one of the windows at the test location. The image was recorded through the other window using a Graphlex camera $\left(4 \times 5^{\prime \prime}\right.$ film format, Polaroid Type 55 film) at a magnification of $6: 1$. The photographs were obtained in a darkened room, varying the time delay between the shock wave passing the downstream pressure transducer and the time of the flash so that various portions of the breakup process could be observed from repeated tests (at least two photographs were obtained for each test condition and delay time).

Pulsed shadowgraph photography was tedious for accumulating data on drop breakup over the wide range of conditions of the present investigation; therefore, the bulk of the results were obtained using motion picture shadowgraphs within a darkened room. This involved using a $20 \mathrm{~W}$ copper vapor laser as the light source (Metalaser Technologies, model 2051, $2 \mathrm{~mJ}$ per pulse, $30 \mathrm{~ns}$ pulse duration) and a $35 \mathrm{~mm}$ drum camera (Cordon Inc., model 351 using Agfa 10E75HDNAH film) to record the images at unity magnification. Prior to measurements, the laser was operated in the continuous pulsing mode to reach proper operating temperatures, and the camera drum was brought to proper speed with the camera shutter closed. Laser operation then was terminated briefly, the camera shutter was opened and the shock tube diaphragm was broken. As the shock wave approached the test location, detected by the pressure transducers, the laser was fired as a high frequency burst (controlled by a Hewlett-Packard model 3314 function generator) to capture the breakup process on the film (laser frequency of $6-8 \mathrm{kHz}$ for 20 pictures). The time between film records was known by monitoring the signal generator frequency with a digital oscilloscope. The film records were analyzed using Gould FD 5000 image display as described subsequently. The procedure was to obtain several (5-14) motion picture shadowgraphs for a particular test condition. The data was then grouped to obtain statistically significant results as ensemble averages. The experimental uncertainties of the various measurements will be taken up when the results are discussed. 


\subsubsection{Holography}

The holocamera and reconstruction systems used to measure drop properties after breakup were similar to those of Ruff et al. (1992). An off-axis arrangement was used with optics providing a 2-3:1 magnification of the hologram image itself over a hologram field that included all the drops generated by secondary breakup. This was coupled with reconstruction optics that allowed drop diameters as small as $25 \mu \mathrm{m}$ to be measured with $5 \%$ accuracy and objects as small as $12-15 \mu \mathrm{m}$ to be observed. The properties of the reconstructed sprays were analyzed using the Gould FD 5000 image display system with a field of view of $1.7 \times 2.0 \mathrm{~mm}$. Various locations in the hologram reconstructions could be observed by traversing the hologram in two directions and the video camera of the display system in the third direction.

Drops and other ellipsoidal objects were sized by measuring their maximum and minimum diameters, $d_{\max }$ and $d_{\min }$, through the centroid of the image. Assuming ellipsoidal shapes, the diameter, $d$, of these objects was taken to the the diameter of a sphere having the same volume, $d^{3}=d_{\min }^{2} d_{\max }$. More irregular objects were sized by finding the area and perimeter of their image and computing the maximum and minimum diameters of an ellipsoid matching these properties: given these parameters, $d$ was found as before. Results at each condition were summed over at least three realizations, considering 150-300 liquid elements, to provide drop size distributions, the mass median diameter (MMD) and the Sauter mean diameter (SMD). Experimental uncertainties generally were dominated by finite sampling limitations because each breakup event only yields a limited number of drops. Within the limitations of the definition of drop sizes, which are difficult to quantify, estimated experimental uncertainties (95\% confidence) of the MMD and SMD are $<40 \%$. The drop size distributions are presented in terms of cumulative volume percentages. Experimental uncertainties ( $95 \%$ confidence) of the cumulative volume percentages were the same as for the MMD and SMD for values in the range 10-80\%-becoming larger outside this range due to sampling limitations at large sizes and resolution limitations at small sizes.

\subsection{Test Conditions}

The test conditions are summarized in table 1. Test drops of water, $n$-heptane, ethyl alcohol, mercury and various glycerol mixtures were used to provide a wide range of liquid properties. The liquid properties listed in table 1 were obtained from Lange (1952), except for the surface tension of glycerol mixtures which were measured in the same manner as Wu et al. (1991). Initial drop diameters were in the range $500-1550 \mu \mathrm{m}$, dictated by the need for measurable drop properties after breakup and the difficulties of producing small drops with very viscous liquids. Ranges of other variables are as follows: $\rho_{\mathrm{L}} / \rho_{\mathrm{G}}$ of $580-12,000$; Oh of $0.0006-4$; We of $0.5-1000$; and $\operatorname{Re}$ of $300-16,000$. Although the full range of $\mathrm{Oh}$ was considered for measurements of deformation and breakup regime transitions and dynamics, measurements to find the outcome of breakup were

Table 1 . Summary of the test conditions $\mathbf{s}^{\mathrm{a}}$

\begin{tabular}{lccccccc}
\hline Liquid & $\begin{array}{c}\rho_{\mathrm{L}} \\
\left(\mathrm{kg} / \mathrm{m}^{3}\right)\end{array}$ & $\begin{array}{c}\mu_{\mathrm{L}} \times 10^{4} \\
(\mathrm{~kg} / \mathrm{ms})\end{array}$ & $\begin{array}{c}\sigma \times 10^{3} \\
(\mathrm{~N} / \mathrm{m})\end{array}$ & $\begin{array}{c}d_{0} \\
(\mu \mathrm{m})\end{array}$ & Oh & We & Re \\
\hline Water & 997 & 8.94 & 70.8 & 1000 & 0.0038 & $0.5-236$ & $340-8250$ \\
n-Heptane & 683 & 3.94 & 20.0 & 500 & 0.0036 & $14-137$ & $720-2270$ \\
Ethyl alcohol & 800 & 16.0 & 24.0 & 1000 & 0.011 & $15-375$ & $1110-6060$ \\
Mercury & 13,600 & 15.0 & 475.0 & 850 & 0.00062 & $10-13$ & $3510-4500$ \\
Solutions of & & & & & & & \\
$\quad$ glycerol (\%) & & & & & & & \\
21 & 1050 & 16.0 & 67.3 & 1200 & 0.0071 & $8-130$ & $1540-6390$ \\
42 & 1105 & 35.0 & 65.4 & 1200 & 0.0120 & $8-136$ & $1530-6580$ \\
63 & 1162 & 108.0 & 64.8 & 1200 & 0.0390 & $1-129$ & $480-6420$ \\
75 & 1195 & 356.0 & 63.8 & 1200 & 0.0990 & $2-128$ & $730-6270$ \\
84 & 1219 & 1000 & 63.2 & 1200 & 0.260 & $1-127$ & $500-6210$ \\
92 & 1240 & 3270 & 62.5 & 1200 & 1.050 & $1-268$ & $530-8330$ \\
97 & 1253 & 8350 & 62.4 & 1500 & 1.700 & $1-205$ & $600-8880$ \\
99.5 & 1260 & 12,500 & 62.0 & 1550 & 3.850 & $1-612$ & $630-15,760$ \\
\hline
\end{tabular}

Air initially at $98.8 \mathrm{kPa}$ and $298 \pm 3 \mathrm{~K}$ in the driven section of the shock tube with shock Mach numbers in the range 1.01-1.24. Properties of the air were taken at normal temperature and pressure: $\rho_{\mathrm{G}}=1.18 \mathrm{~kg} / \mathrm{m}^{3}, \mu_{\mathrm{G}}=18.5 \times 10^{-4} \mathrm{~kg} / \mathrm{ms}$. 
limited to $\mathrm{Oh}<0.1$. The We range includes processes from no deformation into the shear breakup regime that are of interest to processes within dense sprays, but does not reach the catastrophic breakup regime studied by Reinecke \& McKay (1969) and Reinecke \& Waldman (1970). As noted earlier, the $\operatorname{Re}$ range of the present experiments is higher than conditions where gas viscosity plays a strong role in drop drag properties; within the present Re range, the drag of spheres only varies in the range 0.6-0.4 (Faeth 1990; White 1974). Shock Mach numbers were relatively low, 1.01-1.24, so that the physical properties within the uniform flow region were not significantly different from in room air.

\section{RESULTS}

\subsection{Deformation and Breakup Regimes}

The presentation of results will begin with definition of deformation and breakup regime transitions in order to help organize the remainder of the findings. The deformation and breakup regime map, showing transitions as functions of We and Oh similar to Hinze (1955) and Krzeczkowski (1980), is illustrated in figure 1. The present evalution of the onset of breakup (the transition to the bag breakup regime) is essentially identical to the findings of Hinze (1955) and Krzeczkowski (1980) within experimental uncertainties. The present results also agree quite well with the transitions found by Krzeczkowski (1980) to shear breakup and multimode breakup (which Krzeczkowski called transition breakup). In view of the somewhat subjective identifications of breakup regimes and their transitions, this level of agreement is quite satisfying.

The observations of transitions to nonoscillatory and oscillatory deformation illustrated in figure 1 have not been reported before. The present definition of the transition to the nonoscillatory deformation regime was taken to be the condition where the drop deformed so that the ratio of its maximum (cross stream) dimension to its initial diameter was 1.1 , corresponding to a deformation of $10 \%$. Following this transition, there was a range of We at each $\mathrm{Oh}$ where the drop decayed back to a spherical shape, much like an overdamped oscillation, yielding nonoscillatory deformation (defined as conditions where the second peak of the diameter fluctuation involved

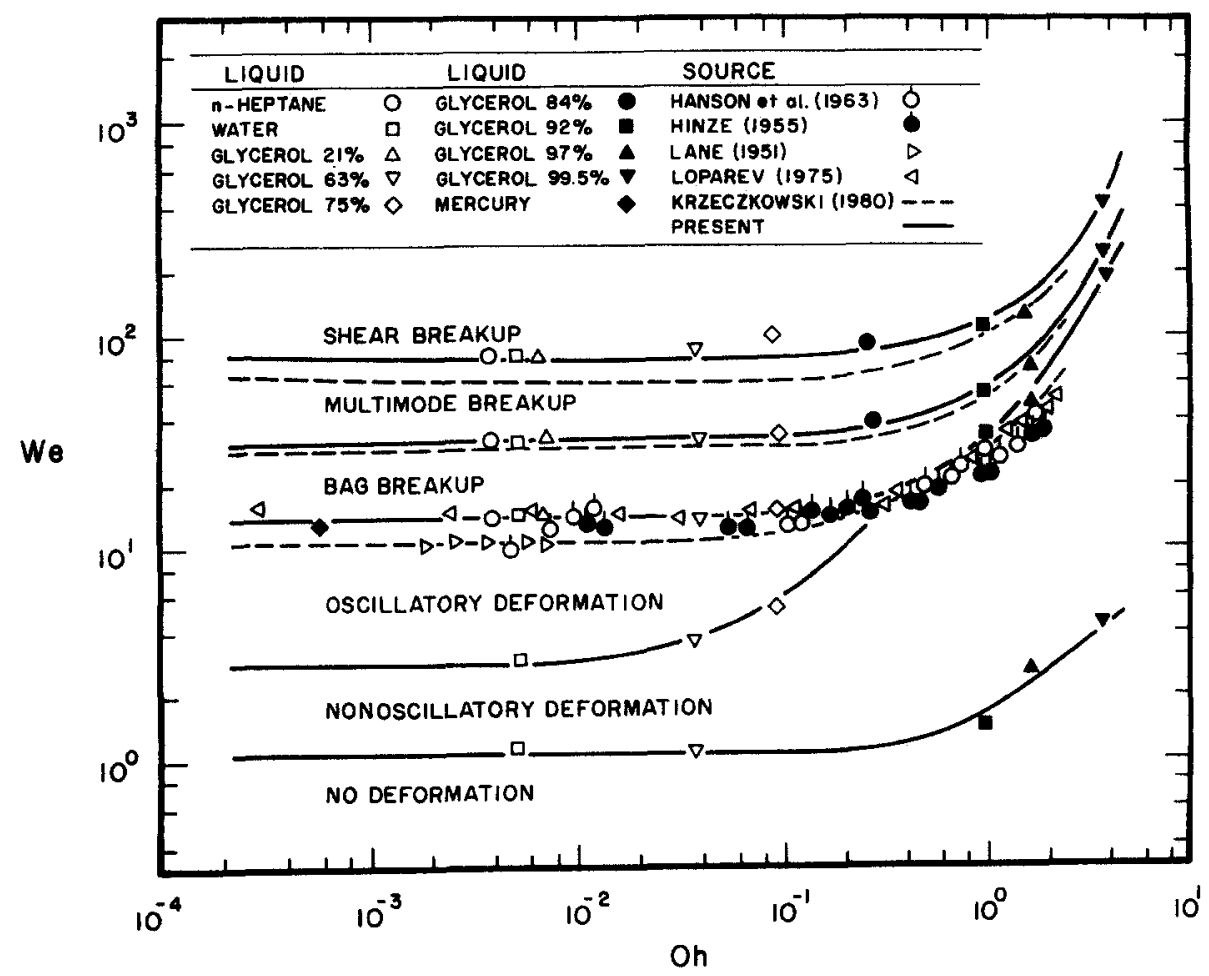

Figure 1. Drop deformation and breakup regime map. 
deformations $<10 \%$ ). For $\mathrm{Oh}>0.4$, this regime was ended by the onset of bag breakup, however, for $\mathrm{Oh}<0.4$, there was a range of We where the drop oscillated with progressively decaying ratios of maximum to initial diameters before the bag breakup regime was reached: this regime is denoted the oscillatory deformation regime in figure 1.

The most striking feature of the flow regime map in figure 1 is that progressively higher We are needed for the various transitions as Oh increases. Hinze (1955) and Krzeczkowski (1980) also noted this effect for the breakup transitions but the behavior is similar for the deformation transitions as well, with the oscillatory deformation regime disappearing entirely for $\mathrm{Oh}>0.4$ as noted earlier. Hinze (1955) concluded that breakup might no longer be observed for $\mathrm{Oh}>2$, however, it appears that $\mathrm{Oh}$ would have to be somewhat greater than 4 , the highest value reached during the present investigation, before breakup would be inhibited for $\mathrm{We}<1000$, with somewhat higher values of $\mathrm{Oh}$ required to inhibit deformation for $\mathrm{We}>1000$.

Recalling that $\mathrm{Oh}$ characterizes the ratio between liquid viscous forces and surface tension forces, the inhibition of deformation and breakup at large Oh clearly is due to increased damping by liquid viscous forces. This slows the deformation process so that drag forces have more time to reduce relative velocities at the point where maximum deformation is reached, and thus the potential for breakup. Another factor is that the final breakup into drops involves Rayleigh type breakup processes which become weak when $\mathrm{Oh}$ is large, so that the drops tend to deform into very long cylindrical threads that exhibit little tendency to divide into drops (at least within the deformation regime). This high $\mathrm{Oh}$ regime is encountered during spray combustion processes at high pressures, where values of surface tension become small but viscosity remains finite as the drop surface nears its thermodynamic critical point. Thus, the findings illustrated in figure 1 suggest that drops at these conditions would not necessarily shatter due to small surface tension as often thought (Faeth 1990); instead, they would deform or even remain spherical. However, additional study of such high pressure drop processes is needed before definitive conclusions about this behavior can be obtained. In particular, specific drop trajectories across the flow regime map depend on the atomization and mixing properties of the spray, while near-critical drop processes involve much lower values of $\rho_{\mathrm{L}} / \rho_{\mathrm{G}}$ than those considered in figure 1 .

All the regime transitions illustrated in figure 1 become relatively independent of liquid viscous forces (or $\mathrm{Oh}$ ) for $\mathrm{Oh}<0.01$. The We for regime transitions in this low $\mathrm{Oh}$ regime are summarized in table 2, considering results from Hinze (1955), Krzeczkowski (1980) and the present study. Similar to the regime map itself, the measurements of the various studies agree within experimental uncertainties. The order of the transitions with increasing We is as follows: nonoscillatory deformation, oscillatory deformation, bag breakup, bag-jet breakup [defined as a separate regime by Krzeckowski (1980) but not during the present study], multimode breakup [which involved evolution from center to edge deformation of the drop and is called transition breakup by Krzeczkowski (1980)] and finally shear breakup. Catastrophic breakup occurs for We $>10^{4}$, which is beyond the present test range.

\subsection{Breakup Times}

The discussion of deformation and breakup regime transitions highlights the importance of breakup times. In particular, as drop velocity relaxation times and breakup times approach one another, the propensity for drop breakup decreases due to reduction of the relative velocities between the drop and the gas. The present measurements of breakup times, along with earlier

Table 2. Summary of We for the transition to deformation and breakup regimes at $\mathrm{Oh}<0.1$

\begin{tabular}{lccc}
\hline Transition to: & $\begin{array}{c}\text { Present } \\
\text { result }\end{array}$ & Krzeczkowski (1980) & Hinze (1955) \\
\hline Nonoscillatory deformation & 1.1 & - & - \\
Oscillatory deformation & 3.0 & - & - \\
Bag breakup & 13 & 10 & 13 \\
Bag-jet breakup & - & 18 & - \\
Multimode breakup & 35 & $30^{\mathrm{a}}$ & - \\
Shear breakup & 80 & 63 & - \\
\hline
\end{tabular}

aCalled the transition regime by Krzeczkowski (1980). 
measurements for shock wave disturbances due to Engel (1958), Simpkins \& Bales (1972), Ranger \& Nicholls (1969), Reinecke \& McKay (1969) and Reinecke \& Waldman (1970) are plotted as a function of We in figure 2 . The breakup times, $t_{b}$, in the figure are normalized by the characteristic breakup time for shear breakup defined by Ranger \& Nicholls (1969) as follows:

$$
t^{*}=d_{0}\left(\rho_{\mathrm{L}} / \rho_{\mathrm{G}}\right)^{1 / 2} / u_{0} .
$$

Except for the present results, which are grouped according to $\mathrm{Oh}$, the measurements are for $\mathrm{Oh}<0.1$ and the effects of liquid viscosity are small. Thus, the deformation and breakup regimes at small $\mathrm{Oh}$ identified in table 2 are illustrated in the figure for reference purposes (omitting catastrophic etc. breakup regimes at high $\mathrm{We}$, as noted earlier).

A remarkable feature of the breakup time results in figure 2 at $\mathrm{Oh}<0.1$ is that $t_{\mathrm{b}} / t^{*}$ varies very little even though We varies over a large range (roughly $10-10^{6}$ ) and a variety of breakup regimes are involved. In fact, the breakup time correlation of Ranger \& Nicholls (1969), developed for the shear breakup regime

$$
t_{\mathrm{b}} / t^{*}=5.0,
$$

provides a reasonably good correlation of all the measurements illustrated in figure 2 for $\mathrm{Oh}<0.1$. However, when the present results for $\mathrm{Oh}>0.1$ are considered, it is seen that $t_{\mathrm{b}} / t^{*}$ progressively increases with increasing $\mathrm{Oh}$. This reflects the importance of liquid viscosity on breakup, evident from the breakup regime map in figure 1 ; in particular, large Oh involves the eventual suppression of breakup so that $t_{\mathrm{b}} / t^{*}$ becomes unbounded. An empirical fit of this behavior over the present test range is as follows:

$$
t_{\mathrm{b}} / t^{*}=5 /(1-\mathrm{Oh} / 7) ; \quad \mathrm{We}<10^{3} .
$$

Equation [3] is seen to provide a reasonable correlation of the present data, however, it is only provisional because it is based on relatively few data with $\mathrm{Oh}$ generally $<3.5$.

\subsection{Drop Deformation}

The first stage of drop deformation, in the period where the drop flattens and first reaches a maximum cross stream dimension, was studied due to its influence on drop velocity relaxation and breakup. In particular, the distortion of the drop should affect its drag properties, and thus relative velocities during the breakup process, which undoubtedly plays a role in the onset of breakup. The

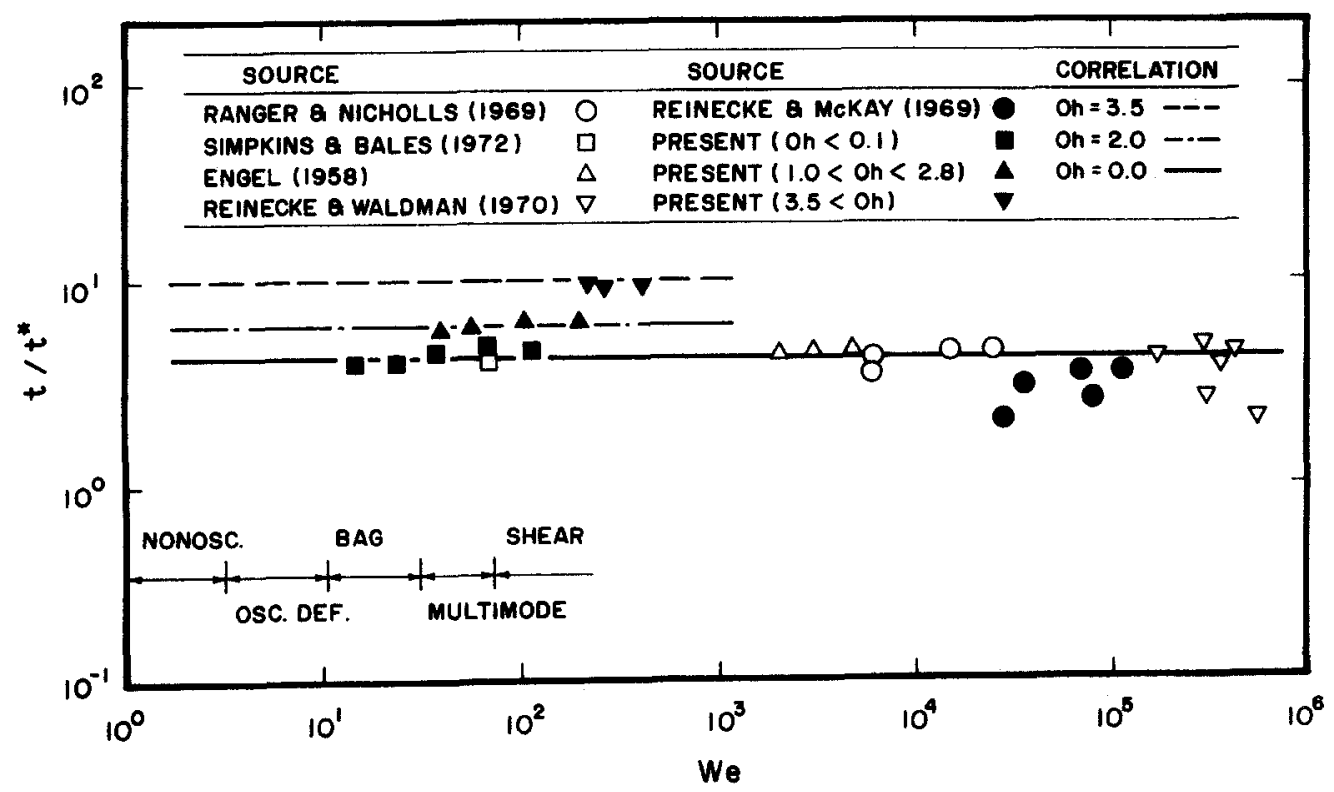

Figure 2. Drop breakup times as a function of We and Oh. 
experimental uncertainties ( $95 \%$ confidence) of the present measurements of drop dimensions in this period are estimated to be $<5 \%$.

The present measurements yielded the cross stream drop diameter, $d_{\mathrm{c}}$, as a function of time, $t$, up to the onset of breakup. The results are plotted as the cross stream distortion, $d_{c}-d_{0}$, normalized by the maximum cross stream distortion, as a function of $t / t *$ in figure 3 (the properties of the maximum distortion will be taken up later). The results of Ranger \& Nicholls (1969), Engel (1958) and Wierzba \& Takayama (1988) for shear breakup $\left(10^{2}<\mathrm{We}<10^{5}\right)$ are shown in the figure along with present results for the deformation and bag breakup regimes, to indicate behavior at the limits of the breakup process. All these results are for $\mathrm{Oh}<0.1$, where the effects of liquid viscosity on breakup times are small.

When normalized in the manner of figure 3, drop distortion correlates reasonably well as a linear function of time. The maximum distortion is reached at roughly $t / t^{*}=1.6$, or at roughly $30 \%$ of the total breakup time. Notably, measurements discussed by Gel'fand et al. (1974) for a similar range of conditions, and plotted by Wierzba \& Takayama (1988) for the shear breakup regime, exhibit very similar behavior. However, the very high $\mathrm{We}\left(>10^{6}\right)$ measurements of Reinecke \& Waldman (1970) exhibit somewhat delayed growth to $d_{\text {cmax }}$. These findings suggest that scaling of drop distortion in the early stages of breakup is relatively universal for $\mathrm{We}<10^{5}$, which includes the deformation, bag breakup and shear breakup regimes: this is in general agreement with the effects of We and breakup regime on the breakup times discussed in connection with figure 2 .

As might be expected, measurements of drop distortion at $\mathrm{Oh}>0.1$, show a progressive delay in the time required for the drop to reach maximum distortion. In fact, this behavior is very similar to the effects of Oh on breakup time, so that results like figure 3 can be obtained in terms of a corrected characteristic breakup time,

$$
t_{\mathrm{c}}^{*}=t^{*} /(1-\mathrm{Oh} / 7) \text {, }
$$

over the present test range (We $<10^{3}, \mathrm{Oh}<3.5$ ).

The next parameter of interest is the maximum cross stream diameter of the drop, $d_{\mathrm{cmax}}$. An approximate expression for the variation of $d_{\mathrm{cmax}}$ with the flow conditions can be obtained for conditions where the effects of liquid viscosity are small, $\mathrm{Oh}<0.1$, by considering the interaction between surface tension and pressure forces when the drop is drawn into a flattened shape. For

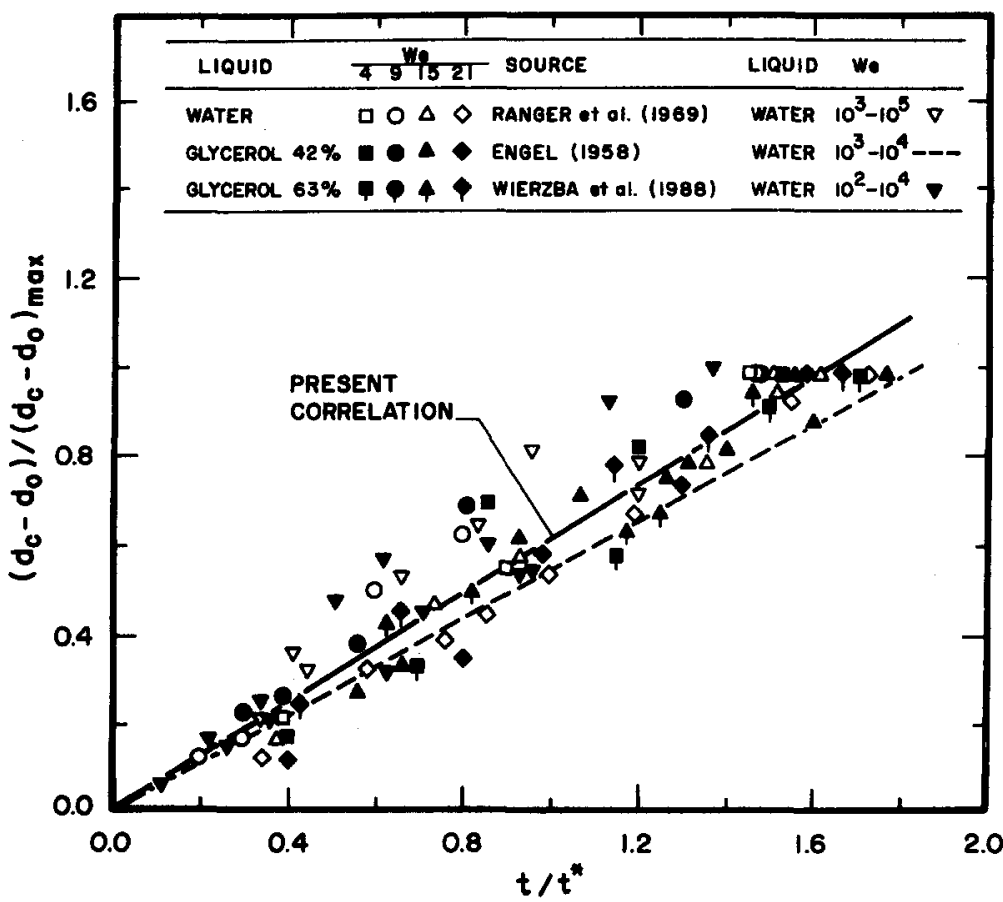

Figure 3. Drop deformation prior to breakup as a function of normalized time. 
this treatment, the following assumptions are made: variations in the relative velocity up to the time $d_{\text {cmax }}$ is reached are neglected; the pressure difference between the bulk of the drop liquid and the region near the edge of the drop is assumed to be proportional to the dynamic head of the flow, $\rho_{\mathrm{G}} u_{0}^{2} / 2$; surface tension forces are assumed to act near the periphery of the deformed (ellipsoidal shaped) drop, along a perimeter of length $\pi d_{\text {cmax }}$ to resist the pressure forces; and the pressure forces are assumed to act across a peripheral cross sectional area $\pi d_{\mathrm{cmax}} d_{\mathrm{smin}}$, where $d_{\mathrm{smin}}$ is the streamwise diameter of the drop along its axis when $d_{\mathrm{cmax}}$ is reached. Equating these forces yields:

$$
2 \sigma \pi d_{\mathrm{cmax}}=C_{\mathrm{f}} \pi d_{\mathrm{cmax}} d_{\mathrm{smin}} \rho_{\mathrm{G}} u_{0}^{2} / 2,
$$

where $C_{\mathrm{f}}$ is an empirical coefficient of order of magnitude unity to allow for the effects of the actual pressure distribution and the shape of the drop. During the period of deformation, the total volume of the drop is conserved; thus, assuming that the deformed drop is an ellipsoid about its flow axis, there results

$$
d_{\mathrm{smin}} d_{\mathrm{cmax}}^{2}=C_{\mathrm{v}} d_{0}^{3}
$$

where $C_{\mathrm{v}}$ is an empirical coefficient of order of magnitude unity to allow for departures of the drop from an ellipsoidal shape. Eliminating $d_{\text {smin }}$ between [5] and [6] then yields

$$
d_{\text {cmax }} / d_{0}=\left(C_{\mathrm{f}} C_{\mathrm{v}} / 2\right)^{1 / 2} \mathrm{We}^{1 / 2} \text {. }
$$

Finally, accounting for the fact that $d_{\text {cmax }} / d_{0}$ approaches unity as We becomes small, and fitting the empirical constant using the present measurements, yields

$$
d_{\text {cmax }} / d_{0}=\left(d_{\text {smin }} / d_{0}\right)^{-1 / 2}=1+0.19 \mathrm{We}^{1 / 2}, \quad \text { Oh }<0.1, \mathrm{We}<10^{2},
$$

where the second part of [8] follows from [5] taking $C_{\mathrm{v}}=1$ (which was representative of the present measurements).

Figure 4 is an illustration of the present measurements of $d_{\text {cmax }} / d_{0}$ as a function of We, with $\mathrm{Oh}$ as a parameter. The correlating expression of [8] for $\mathrm{Oh} \leqslant 0.1$ also is plotted in the figure. It is evident that [8] provides a reasonable fit of the data, however, it should be noted that [8] is slightly inconsistent with the transition to the nonoscillatory deformation regime of figure 1 because it somewhat overestimates $d_{\text {cmax }} / d_{0}$ near $\mathrm{We}=1$ (by roughly $10 \%$ ). The effects of increasing Oh can be seen, with $d_{\text {cmax }} / d_{0}$ tending to decrease at a particular We as Oh is increased. Because the deformation motions of the drop cease at the point where $d_{\mathrm{cmax}}$ is reached, this behavior is not thought to be a direct effect of viscous forces on the force balance fixing $d_{\text {cmax }}$. Instead, the increased time of deformation due to the effects of liquid viscosity is a more probable mechanism. This allows drag forces to act for a longer time before the maximum deformation condition is reached, which tends to reduce the realtive velocity, and correspondingly $d_{\text {cmax }}$ through [5] and [6]. This effect also must be responsible for the increased We required for transition to the nonoscillatory deformation

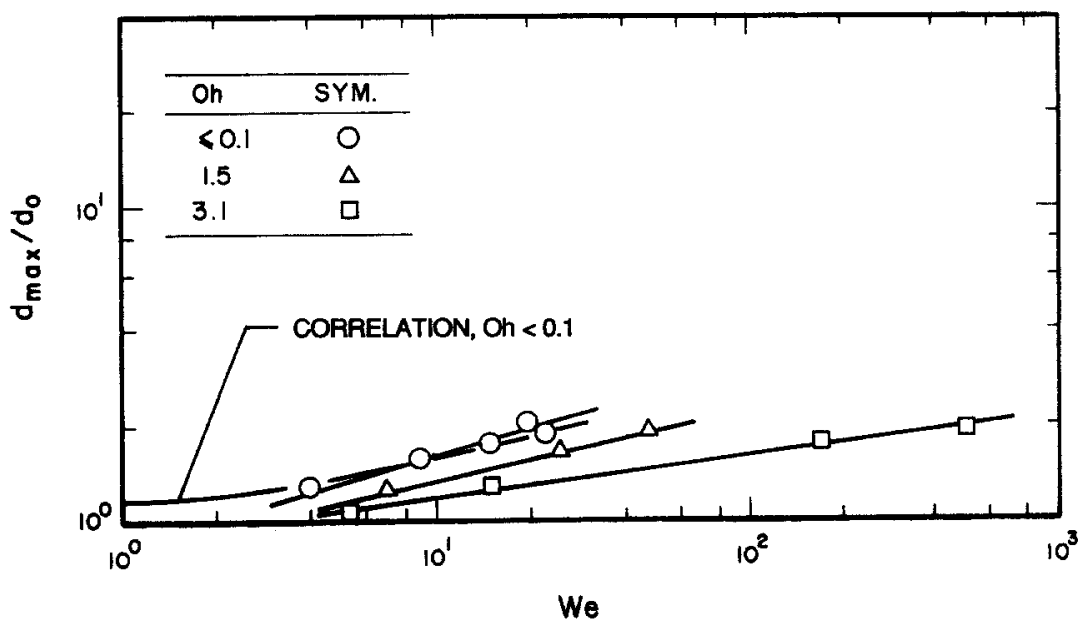

Figure 4. Maximum cross stream drop diameters prior to breakup as a function of We and Oh 
regime as $\mathrm{Oh}$ increases, seen in figure 1 . To initiate work toward quantifying this mechanism, the drag properties of drops as they deform will be taken up next.

\subsection{Drop Drag}

Drop drag properties were found by measuring the motion of the centroid of the drop in the uniform flow field behind the shock wave. This approach is only approximate because it neglects the forces involved as the mass of the drop is redistributed during drop deformation. However, this effect is not expected to be large for the present test conditions because the characteristic velocities in the liquid phase are small. For example, considering either the normal motion of liquid along the axis due to the static pressure increase near the forward stagnation point, or the acceleration of the liquid as the local static pressure decreases in moving toward the edge of the deformed drop, yields the following characteristic liquid phase velocity:

$$
u_{\mathrm{L}}=\left(\rho_{\mathrm{G}} / \rho_{\mathrm{L}}\right)^{1 / 2} u_{0} \text {. }
$$

For the present conditions $u_{\mathrm{L}} / u_{0}$ is in the range $0.03-0.04$, so that the motion of the drop as a whole should dominate the drag properties. Additionally, pressure gradient forces are negligible because the flow behind the shock wave is uniform, and virtual mass and Basset history forces can be neglected because $\rho_{\mathrm{L}} / \rho_{\mathrm{G}} \gg 1$ for the present test conditions (Faeth 1987).

The drop drag coefficient, $C_{\mathrm{D}}$, was defined in terms of the local relative velocity, $u$, and cross stream dimension of the drop as follows:

$$
C_{\mathrm{D}}=D /\left(\pi d_{\mathrm{c}}^{2} \rho_{\mathrm{G}} u^{2} / 8\right),
$$

where $D$ is the drag force on the drop. Under the present assumptions only the acceleration of the drop must be considered when evaluating the drag force, yielding the following expression for $C_{\mathrm{D}}$ from the measurements of the centroid position, $x$, as a function of time:

$$
C_{\mathrm{D}}=2 \rho_{\mathrm{L}} d_{0}^{3} \mathrm{~d}^{2} x / \mathrm{d} t^{2} /\left[3 \rho_{\mathrm{G}} d_{\mathrm{c}}^{2}\left(u_{0}-\mathrm{d} x / \mathrm{d} t\right)^{2}\right] .
$$

The measurements of $C_{\mathrm{D}}$ primarily were limited by the accuracy of defining centroid motion at small times after passage of the shock wave, to yield experimental uncertainties ( $95 \%$ confidence) $<30 \%$.

The experiments to find $C_{\mathrm{D}}$ involved the initial deformation of the drops up to the time $d_{\mathrm{cmax}}$ was reached, Oh $<0.1$ and a moderate range of $\operatorname{Re}(1000-2500)$, where the effects of $\operatorname{Re}$ on the drag of the drops are expected to be small (Faeth 1987). Thus, it was found that $C_{\mathrm{D}}$ largely was a function of the degree of deformation of the drop for the present test conditions. In order to highlight this behavior, the results are plotted in terms of $d_{\mathrm{c}} / d_{0}$ in figure 5. Measurements of $C_{\mathrm{D}}$ for solid spheres and thin disks, drawn from White (1974) for the same range of Re as the present tests, also are illustrated in the plot. In spite of the relatively large uncertainties of the measurements, the trend of the data is quite clear; for $d_{\mathrm{c}} / d_{0}$ near unity, $C_{\mathrm{D}}$ approximates results for solid spheres and then increases to approach results for thin disks at $d_{\mathrm{c}} / d_{0} \approx 2$. Thus, behavior in the period observed appears to be dominated by distortion of the drop, rather than internal circulations which would cause reductions of $C_{D}$ from values appropriate for solid spheres. This seems reasonable because the characteristic liquid phase velocities are relatively small for the present test conditions, $\mathrm{cf}$. [9].

\subsection{Drop Sizes}

Measurements of drop sizes after breakup were limited to conditions where $\mathrm{Oh}<0.1$. This was necessary in order to capture the entire drop field after breakup on a single hologram, because larger values of $\mathrm{Oh}$ yielded regions containing drops that were too large for the present optical arrangement. The measurements included $\mathrm{We}<10^{3}$, which corresponds to the bag, transition and shear breakup regimes.

Past work on the structure of dense sprays and processes of primary breakup of nonturbulent and turbulent liquids (Ruff et al. 1992; Wu et al. 1991, 1992), indicated that local drop size distributions generally satisfied the universal root normal distribution function of Simmons (1977), with MMD/SMD = 1.2 [see Belz (1973) for a discussion of the properties of the root normal distribution function]. This vastly simplifies the presentation of data because the root normal 


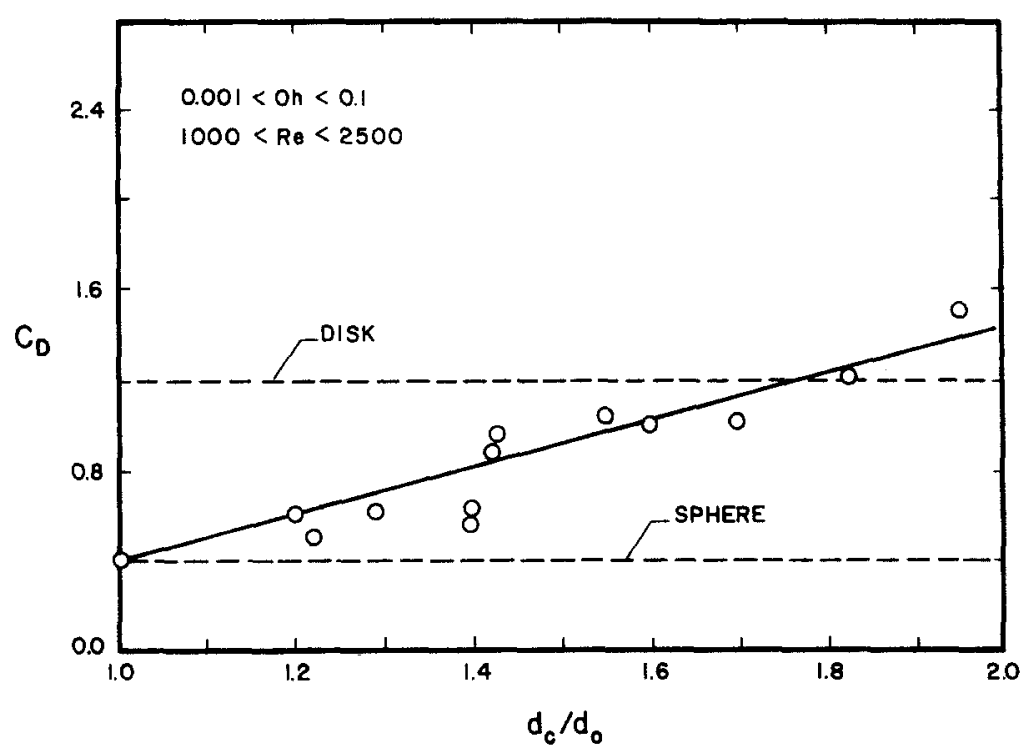

Figure 5. Drop drag coefficient prior to breakup as a function of the normalized cross stream diameter.

distribution only has two moments, and with MMD/SMD a constant, the distribution is entirely specified by the SMD alone. Thus, initial measurements of drop sizes after breakup focused on evaluating the root normal distribution function. This included tests with water, 42 and $63 \%$ glycerol mixtures, $n$-heptane and ethyl alcohol for We in the range 15-375.

Typical results of the drop size distribution measurements are illustrated in figure 6 for bag breakup, figure 7 for multimode breakup and figure 8 for shear breakup. The results are plotted in terms of the root normal distribution function, with the function itself illustrated for values of $\mathrm{MMD} / \mathrm{SMD}=1.1,1.2$ and 1.5. The data are somewhat scattered at large drop sizes because the number of large drops is limited by the breakup of single drops. The results for the bag and multimode breakup regimes (figures 6 and 7) are represented reasonably well by the universal root

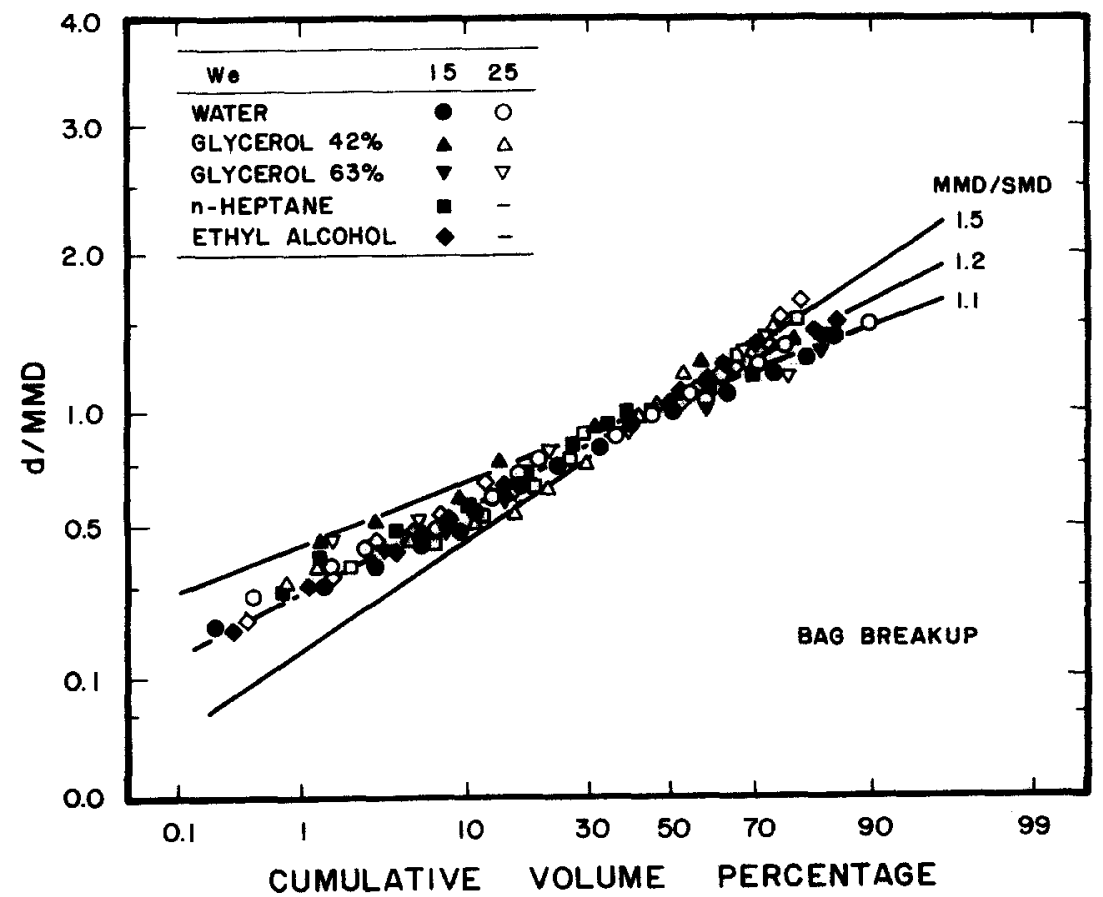

Figure 6. Distribution of drop diameters after bag breakup. 


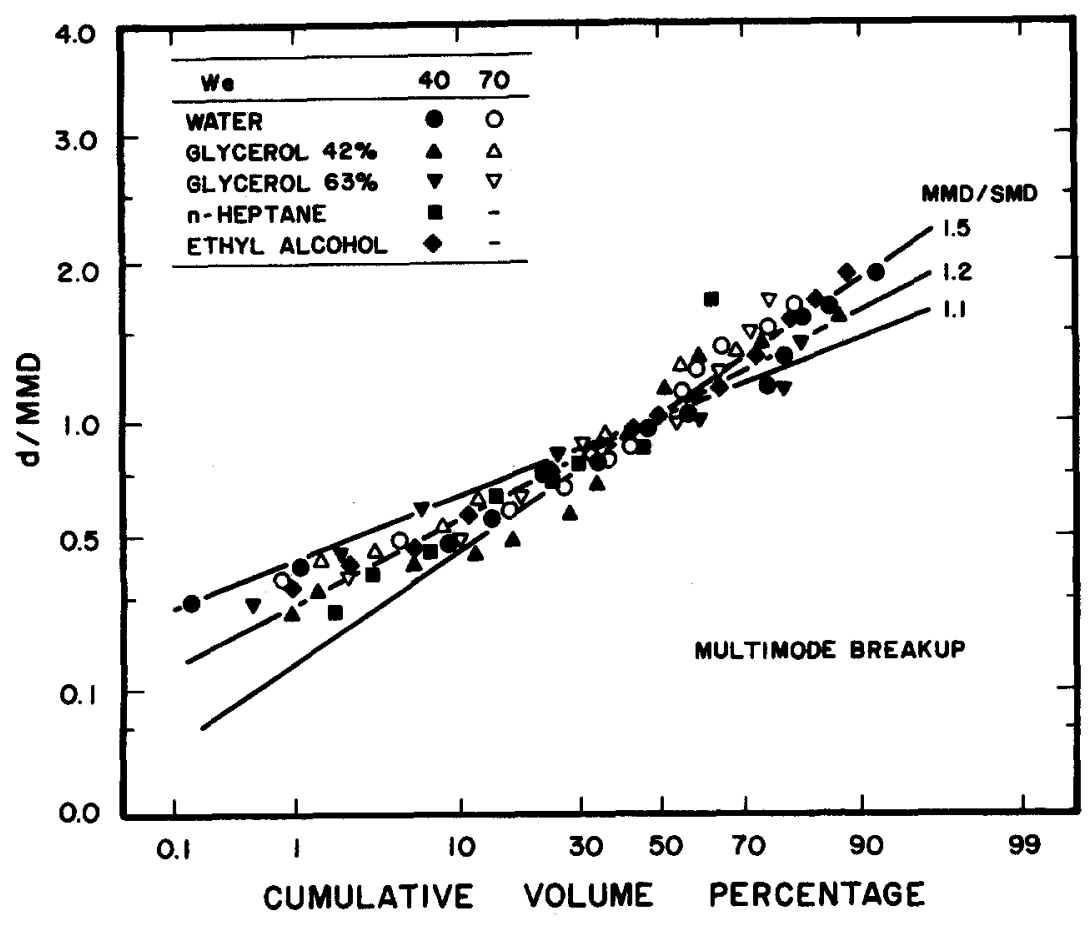

Figure 7. Distribution of drop diameters after multimode breakup.

normal distribution function with $\mathrm{MMD} / \mathrm{SMD}=1.2$, similar to the findings of Ruff et al. (1992) for the near-injector (dense) spray region for pressure atomization and Wu et al. $(1991,1992)$ for primary breakup of nonturbulent and turbulent liquids. In contrast to the present findings, Gel'fand et al. (1974) observed a bimodal distribution of drop sizes after bag breakup for the two conditions they considered. The reason for this discrepancy is unknown and clearly merits additional study.

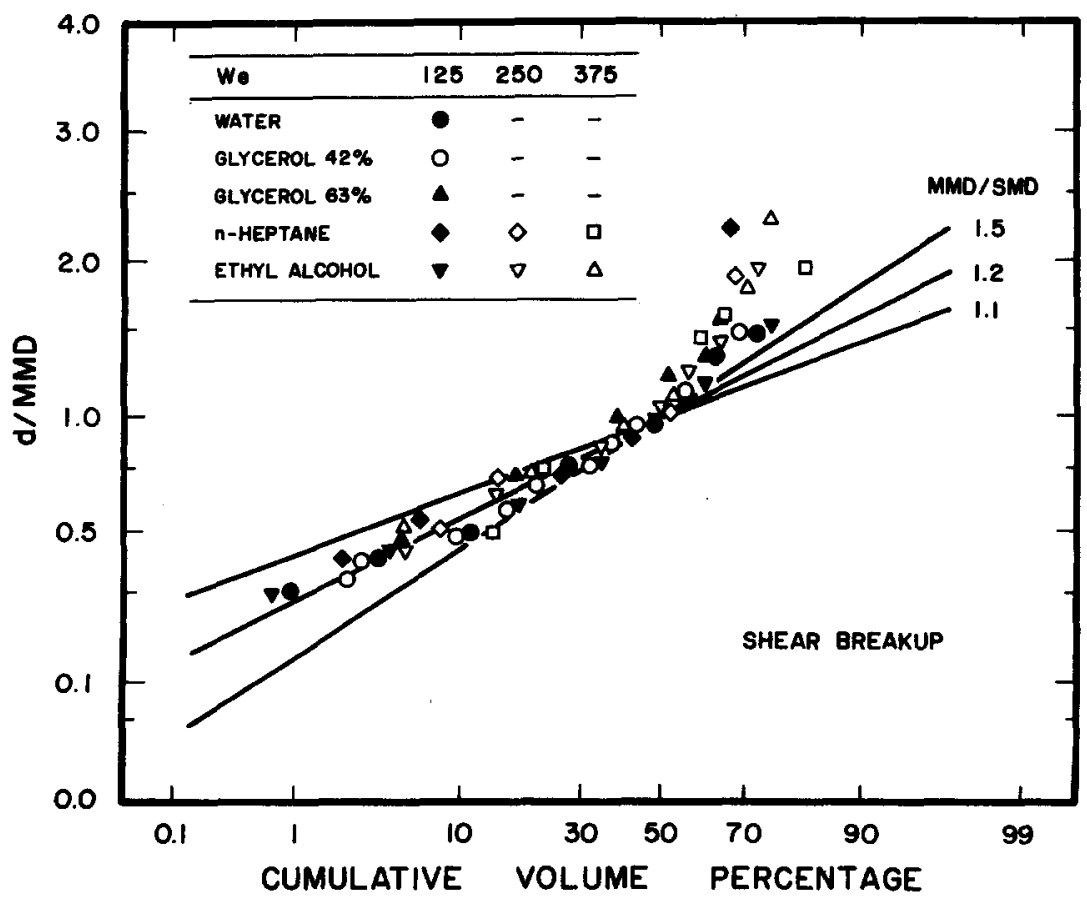

Figure 8. Distribution of drop diameters after shear breakup. 
The present results yielded bimodal behavior for shear breakup (figure 8) where departure from the root normal drop size distribution for drop sizes greater than the MMD is clearly evident. This behavior was caused by the core (or drop-forming) drop that remained after the stripping of smaller drops from its periphery ceased. Conditions for ending stripping involved a combination of reduced drop sizes and reduced relative velocities so that a description of the behavior was not possible without more information on drop velocities during and after breakup. Results thus far, however, indicate that the universal root normal distribution is only effective for bag and multimode breakup where several large drops form from the ring at the base of the bag, rather than the single core drop of the shear breakup process. This is consistent with observations of Ruff et al. (1992) that the universal root normal distribution was effective for dense sprays, including conditions following secondary breakup, because secondary breakup in the bag and multimode regimes dominated their test conditions.

A correlating expression for the SMD after secondary breakup can be obtained by noting the similarity between primary breakup of nonturbulent liquids and shear breakup of drops. In both cases, drops or ligaments are stripped from boundary layers in the liquid phase that form near the liquid surface: on the windward side of waves along the surface for primary breakup of nonturbulent liquids (Wu et al. 1991); and on the windward side of the drop for secondary breakup in the shear breakup regime. The configuration for secondary breakup in the shear breakup regime is illustrated in figure 9, where the core (drop-forming) drop is illustrated. It is assumed that the relative velocity at the time of breakup can be represented by the initial relative velocity, $u_{0}$, and that drop sizes after breakup are comparable to the thickness, $\delta$, of the boundary layer as it reaches the periphery of the drop. Since this boundary layer develops while moving away from the forward stagnation point of the flow, the characteristic velocity in the liquid phase is taken to be $u_{\mathrm{L}}$ from [9]. Additionally, the SMD is dominated by the largest drop sizes in the distribution so that the length of development of the liquid boundary layer is taken to be proportional to $d_{0}$, which should be the condition tending to yield the largest drop sizes. Finally, assuming that the boundary layer is laminar, due to the relatively small values of $u_{\mathrm{L}}$ and $d_{0}$, there results

$$
\operatorname{SMD} / d_{0}=C_{\mathrm{s}}\left(\rho_{\mathrm{L}} / \rho_{\mathrm{G}}\right)^{1 / 4}\left[\mu_{\mathrm{L}} /\left(\rho_{\mathrm{L}} d_{0} u_{0}\right)\right]^{1 / 2}
$$

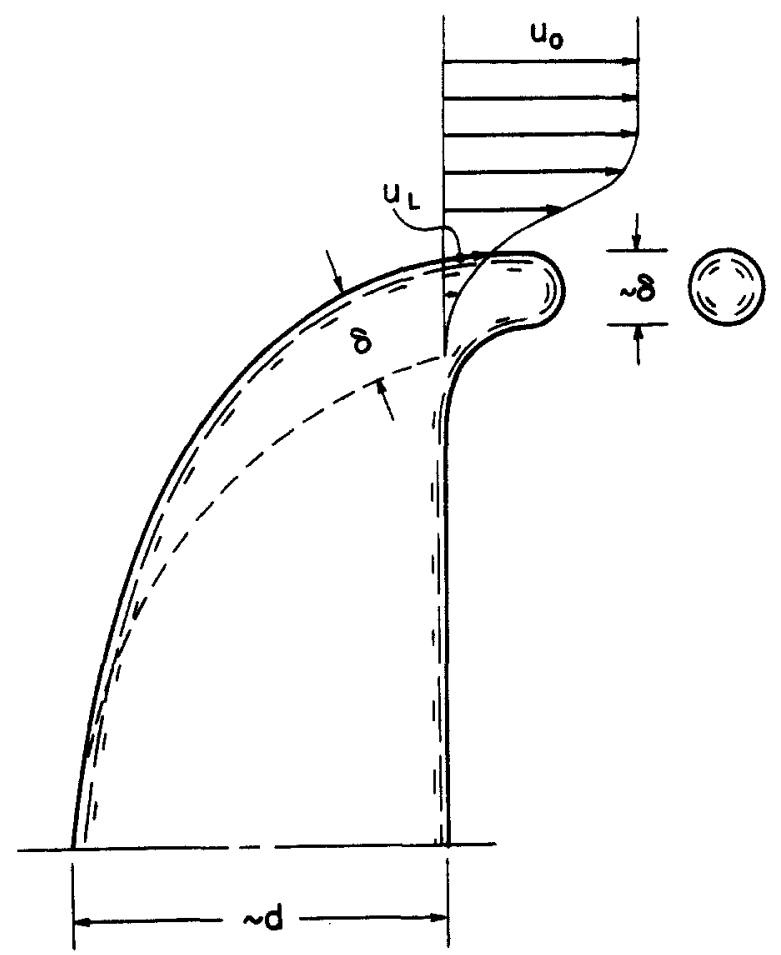

Figure 9. Sketch of the shear breakup process. 
where $C_{\mathrm{s}}$ is an empirical constant involving the various proportionality factors. It is convenient to rearrange [12] so that the We based on the SMD is obtained because this helps assess the potential for subsequent breakup of the largest drops in the distribution. Completing this rearrangement yields:

$$
\rho_{\mathrm{G}} \mathrm{SMD} u_{0}^{2} / \sigma=C_{\mathrm{s}}\left(\rho_{\mathrm{L}} / \rho_{\mathrm{G}}\right)^{1 / 4}\left[\mu_{\mathrm{L}} /\left(\rho_{\mathrm{L}} d_{0} u_{0}\right)\right]^{1 / 2} \mathrm{We} .
$$

The present measurements of the SMD after secondary breakup are plotted in terms of [13] in figure 10, considering the same liquids as figures 6-8. These results are for $\mathrm{Oh}<0.1$ and $\mathrm{We}<10^{3}$, including the bag, transition and shear breakup regimes. A correlation of the data according to [13] is also shown in the plot; the power of this correlation is unity within experimental uncertainties, yielding the following empirical fit:

$$
\rho_{\mathrm{G}} \mathrm{SMD} u_{0}^{2} / \sigma=6.2\left(\rho_{\mathrm{L}} / \rho_{\mathrm{G}}\right)^{1 / 4}\left[\mu_{\mathrm{L}} /\left(\rho_{\mathrm{L}} d_{0} u_{0}\right)\right]^{1 / 2} \mathrm{We} .
$$

The standard deviations of the coefficient and the overall factor on the right-hand side of [14] are 20 and $10 \%$, respectively, with the correlation coefficient of the fit being 0.91 . It should be noted, however, that $\rho_{\mathrm{L}} / \rho_{\mathrm{G}}$ does not vary greatly over the present test range and additional measurements are needed to explore density ratio effects.

It is probably fortuitous, and certainly surprising, that a single correlation can express the SMD after bag, multimode and shear breakup. In particular, the three breakup mechanisms appear to be rather different, while the drop size distribution after shear breakup differs from the other two breakup regimes (cf. figures 6-8). On the other hand, similar behavior for the three breakup regimes is consistent with the observation that their breakup times correlate in the same way, as discussed in connection with figure 2. Additionally, the largest drops formed during bag breakup come from the ring at the base of the bag, which has length and velocity scales during its formation that are

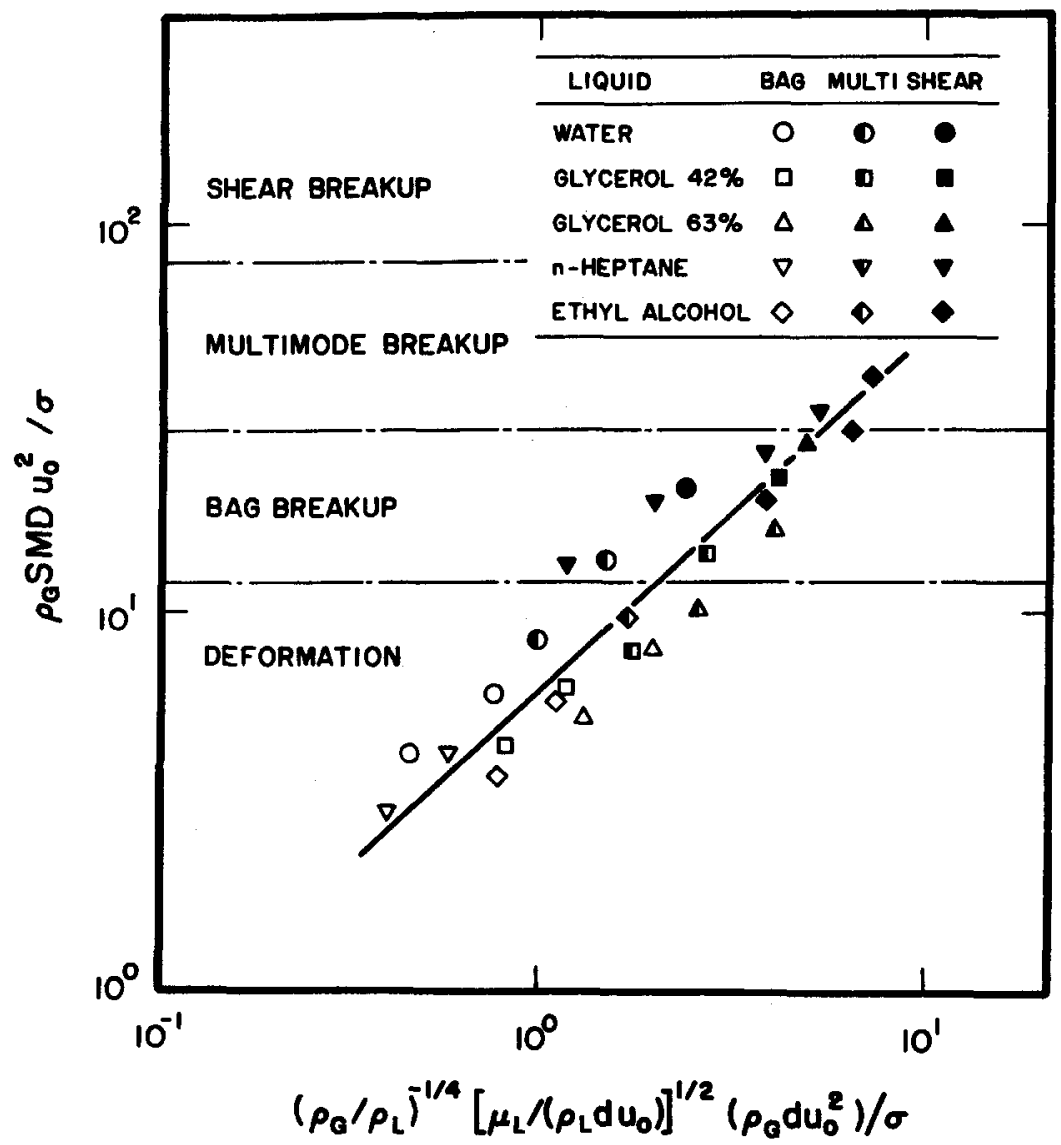

Figure 10. Correlation of the SMD after secondary breakup. 
similar to those of shear breakup. This supports the similarity of the SMD after breakup for the bag and shear breakup regimes, with related behavior for the multimode breakup regime that separates them.

Because the largest drops after secondary breakup dominate the SMD, it is of interest to consider their potential for subsequent breakup and the roles that reduced drop sizes and relative velocities play in ending the breakup process. In order to assess the potential for additional deformation and breakup, the regime transitions at low $\mathrm{Oh}$ from table 2 have been drawn in figure 10 (interpreting the ordinate as the We of particular drops in the distribution and assuming that $u_{0}$ is still representative of the relative velocity). Noting that more than half the mass of the spray involves drop diameters greater than the SMD (recall that typically MMD/SMD $=1.2$ ), it is clear that a significant fraction of the drops after secondary breakup would be in the deformation and bag breakup regimes if the relative velocities of the large drops still approximated $u_{0}$. For the present test conditions, however, there was no evidence of subsequent breakup of large drops, even though the breakup times of these drops are shorter than the original drop, cf. [1] and [2]. Thus, it is likely that reduction of the relative velocity during breakup is an important factor in stabilizing large drops after secondary breakup, along with the effect of reduced drop diameters. Measurements of drop velocities during and after secondary breakup clearly are needed to better understand how secondary breakup ends; therefore, work along these lines has been initiated in this laboratory.

\section{CONCLUSIONS}

Drop deformation and secondary breakup after a shock wave initiated disturbance were studied, considering drops of water, $n$-heptane, ethyl alcohol, mercury and various glycerol mixtures in air at normal temperature and pressure (We of $0.5-1000$; Oh of $0.0006-4 ; \rho_{\mathrm{L}} / \rho_{\mathrm{G}}$ of $580-12,000$; and $\operatorname{Re}$ of $300-16,000)$. The major conclusions of the study are as follows:

1. Drop deformation and breakup occurs at $\mathrm{We}>1$, with the following deformation and breakup regimes identified (listed in order of appearance with increasing We at $\mathrm{Oh}<0.1)$ : no deformation, nonoscillatory deformation, oscillatory deformation, bag breakup, multimode breakup and shear breakup. The We for the onset of deformation and breakup regimes increases with increasing Oh, with no breakup observed over the present test range for $\mathrm{Oh}>4$ due to the stabilizing effect of the liquid viscosity.

2. Unified temporal scaling of deformation and breakup processes was observed in terms of a characteristic breakup time that was nearly independent of We and tended to increase with increasing Oh, cf. [1] and [4].

3. Drop drag coefficients evolved from the properties of spheres to those of thin disks as drop deformations progressed prior to breakup.

4. Drop size distributions after secondary breakup satisfied the universal root normal distribution function (Simmons 1977), with $\mathrm{MMD} / \mathrm{SMD}=1.2$ for the bag and multimode breakup regimes, similar to recent observations of drop sizes in pressure-atomized sprays and after primary breakup (Ruff et al. 1992; Wu et al. 1991, 1992), and can be characterized by a single parameter like the SMD. In contrast, drop sizes after shear breakup did not satisfy this distribution function because the largest drops were dominated by the core (drop-forming) drop.

5. Drop sizes after secondary breakup decreased as We increased and could be correlated in a manner similar to recent results for primary breakup of nonturbulent liquids (Wu et al. 1991), i.e. in terms of a characteristic liquid boundary layer thickness for all three breakup regimes, cf. [14]. Drop properties after secondary breakup at high We suggest potential for the subsequent breakup of the largest drops in the size distribution if relative velocities did not change during breakup; thus, the fact that the largest drops were observed to be stable suggests that reductions in both drop sizes and relative velocities play a role in ending the secondary breakup process. 
Conclusions about the outcome of secondary breakup are limited to conditions where $\mathrm{Oh}<0.1$ and additional study at higher $\mathrm{Oh}$ is needed. In addition, practical sprays often involve lower values of $\rho_{\mathrm{L}} / \rho_{\mathrm{G}}$ and $\operatorname{Re}$ than the present experiments and the anticipated effects of modifying these variables should be quantified.

Acknowledgements - This research was sponsored by the Air Force Office of Scientific Research, Grant No. 89-0516, under the technical management of J. N. Tishkoff. The authors also would like to thank C. W. Kauffman for the loan of the shock tube facility and advice concerning its operation. The U.S. Government is authorized to reproduce and distribute copies for governmental purposes notwithstanding any copyright notation thereon.

\section{REFERENCES}

Belz, M. H. 1973 Statistical Methods in the Process Industries, pp. 103-104. Wiley, New York. Borisov, A. A., Gel'fand, B. E., Natanzon, M. S. \& Kossov, O. M. 1981 Droplet breakup regimes and criteria for their existence. Inzh.-Fiz. Zh. 40, 64-70.

DABORA, E. K. 1967 Production of monodisperse sprays. Rev. Scient. Instrum. 38, 502-506.

ENGEL, O. G. 1958 Fragmentation of waterdrops in the zone behind an air shock. J. Res. Natn. Bur. Stand. 60, 245-280.

FAETH, G. M. 1987 Mixing, transport and combustion in sprays. Prog. Energy Combust. Sci. 13, 293-345.

FAETH, G. M. 1990 Structure and atomization properties of dense turbulent sprays. In Proc. 23rd Symp. (Int.) on Combustion, The Combustion Inst., Pittsburgh, PA, pp. 1345-1352.

Gel'fand, B. E., Gubin, S. A. \& Kogarko, S. M. 1974 Various forms of drop fractionation in shock waves and their special characteristics. Inzh.-Fiz. Zh. 27, 119-126.

GifFen, E. \& Muraszew, A. 1953 The Atomization of Liquid Fuels. Chapman \& Hall, London.

HAAS, F. C. 1964 Stability of droplets suddenly exposed to a high velocity gas stream. $A I C h E J l$ 10, 920-924.

Hanson, A. R., Domich, E. G. \& ADAms, H. S. 1963 Shock-tube investigation of the breakup of drops by air blasts. Phys. Fluids 6, 1070-1080.

HASSLER, G. 1972 Untersuchung zur zerstörung von wassertropfen durch aerodynamische krafte. Forsch. Geb. IngWes. 38, 183-192.

HINZE, J. O. 1955 Fundamentals of the hydrodynamic mechanism of splitting in dispersion processes. AIChE Jl 1, 289-295.

KRZECZKOWSKI, S. A. 1980 Measurement of liquid droplet disintegration mechanisms. Int. J. Multiphase Flow 6, 227-239.

LANE, W. R. 1951 Shatter of drops in streams of air. Ind. Engng Chem. 43, 1312-1317.

LANGe, N. A. 1952 Handbook of Chemistry, 8th edn, pp. 1134 \& 1709. Handbook Publishers, Sandsuky, $\mathrm{OH}$.

Liang, P. Y., EASTES, T. W. \& GhaRAKHARI, A. 1988 Computer simulations of drop deformation and drop breakup. AIAA paper No. 88-3142.

LOPAREV, V. P. 1975 Experimental investigation of the atomization of drops of liquid under conditions of a gradual rise of the external forces. Izv. Akad. Nauk SSSR Mekh. Zhidkosti Gaza 3, 174-178.

RaNGer, A. A. \& Nicholls, J. A. 1969 The aerodynamic shattering of liquid drops. AIAA $J l$ 7, 285-290.

REINECKE, W. G. \& MCKAY, W. L. 1969 Experiments on waterdrop breakup behind Mach 3 to 12 shocks. Sandia Corp. Report SC-CR-70-6063.

REINECKE, W. G. \& WALDMAN, G. D. 1970 A study of drop breakup behind strong shocks with applications to flight. Avco Report AVSD-0110-70-77.

RufF, G. A., Wu, P.-K., Bernal, L. P. \& Faeth, G. M. 1992 Continuous- and dispersed-phase structure of dense nonevaporating pressure-atomized sprays. J. Prop. Power 8, 280-289.

SANGiovanNi, J. \& KESTIN, A. S. 1977 A theoretical and experimental investigation of the ignition of fuel droplets. Combust. Sci. Technol. 16, 59-70. 
Simmons, H. C. 1977 The correlation of drop-size distributions in fuel nozzle sprays. J. Engng Power 99, 309-319.

Simpkins, P. G. \& Bales, E. J. 1972 Water-drop response to sudden accelerations. J. Fluid Mech. $55,629-639$.

VolynSKII, M. S. \& LIPATov, A. S. 1970 Deformation and disintegration of liquid drops in a gas flow. Inzh.-Fiz. Zh. 18, 838-843.

White, F. M. 1974 Viscous Fluid Flow. McGraw-Hill, New York.

WIERZBA, A. \& TAKAYAMA, K. 1988 Experimental investigation of the aerodynamic breakup of liquid drops. AIAA $J l$ 26, 1329-1335.

Wu, P.-K., RufF, G. A. \& FAETH, G. M. 1991 Primary breakup in liquid/gas mixing layers. Atomiz. Sprays 1, 421-440.

Wu, P.-K., Tseng, L.-K. \& FaEth, G. M. 1992 Primary breakup in gas/liquid mixing layers for turbulent liquids. Atomiz. Sprays. In press. 